\title{
Short-term antioxidant diet prevents hyperfiltration in young male rat kidney subjected to ischemia/reperfusion injury
}

\author{
Yuriy Slyvka $^{1}$, Felicia V. Nowak ${ }^{1,2}$, Tracey M. Hayes ${ }^{1,2}$, Sharon R. Inman ${ }^{1,2}$ \\ ${ }^{1}$ Department of Biomedical Sciences, Ohio University, Athens, USA \\ ${ }^{2}$ Program in Molecular and Cellular Biology, Ohio University, Athens, USA \\ Email: inmans@ohio.edu
}

Received 16 January 2013; revised 16 February 2013; accepted 23 February 2013

\begin{abstract}
Objectives: The process of transplantation is associated with exposure to both long and short cold and warm ischemic times that result in ischemia/reperfusion injury. Oxidative stress contributes to tissue fibrosis, renal dysfunction, and/or rejection. Treatments that scavenge oxygen free radicals and have antioxidant properties can ameliorate the damaging results in renal grafts following ischemia/reperfusion injury. The present study tests the hypothesis that an antioxidant-fortified diet given to rats before and after renal ischemia/reperfusion injury will reduce the kidney damage that results and improve renal function. Endothelial and inducible nitric oxide synthases may change with tissue injury, including ischemia/ reperfusion. Materials and Methods: Male Wistar rats were subjected to ischemia/reperfusion injury at 7 or 19 weeks of age with or without dietary antioxidant supplementation. One week later, glomerular filtration rate, mean arterial pressure and urinary nitric oxide were measured, and renal endothelial and inducible nitric oxide synthases examined. Results: The glomerular filtration rate was elevated more than two-fold above the normal range at 8 weeks in animals on the regular diet exposed to ischemia/reperfusion, while in the 8 week antioxidant-fortified diet group the glomerular filtration rate was normal. Also, in 8 week rats, levels of endothelial nitric oxide synthase protein in cortex were higher on the regular than on the antioxidant-fortified diet. Conclusion: Early after ischemia/reper-fusion injury renal endothelial nitric oxide synthase levels rise, possibly contributing to vascular dilation and hyperperfusion, and an antioxidant-fortified diet can ameliorate these changes in the younger age group.
\end{abstract}

Keywords: Antioxidants; Ischemia/Reperfusion; Kidney; Nitric Oxide Synthase

\section{INTRODUCTION}

Renal transplantation is the most effective and cost efficient treatment for patients with end stage renal disease [1]. The number of patients requiring kidney transplants in the United States is growing, thus leading to the use of "marginal" kidneys, such as kidneys from older donors being transplanted [2]. Clinical studies have shown that kidneys from donors over the age of 60 do not result in significantly higher serum creatinine levels compared to those in patients receiving much younger donor kidneys when followed for 12 months [3]. In addition, with the "baby boomers" coming of age and due to longer life spans, older recipients are increasingly being considered as renal transplant candidates [4].

The process of transplantation is associated with exposure to both long and short cold and warm ischemic times that result in ischemia/reperfusion (I/R) injury. I/R injury is a non-immune factor that contributes to both short- and long-term graft dysfunction [5,6]. With the growing number of older kidneys being transplanted, this may become an even larger issue both in short- and long-term graft survival. With increasing age, donor kidneys are likely to harbor greater amounts of ischemic tissue and thus the transplanted kidney may be more susceptible to the more severe damaging effects of $I / R$ injury [7]. In conjunction with this, older donor kidneys have fewer functional nephrons compared to younger donor kidneys accounting for a stronger chance of eventual graft dysfunction/rejection [8].

There have been many mechanisms described that are thought to contribute to I/R injury. One such possibility is oxidative stress. Following the $\mathrm{I} / \mathrm{R}$ event, oxygen free radicals are released which are very damaging to the kidney. This causes tissue fibrosis, renal dysfunction, and/or rejection [9-11]. Further, it has been shown that administering treatments that scavenge oxygen free radicals and have antioxidant properties can ameliorate the 
damaging results in renal grafts following $\mathrm{I} / \mathrm{R}$ injury [12-15].

Oxidative stress increases with age. Also, in older rats (21 months), renal tubular dopamine receptors are decreased while the angiotensin II receptors have exaggerated functions which affect sodium homeostasis and blood pressure [16]. In addition, in the aging kidney, cell senescence associated with oxidative stress increases [17]. Thus, both I/R injury and aging cause oxidative stress to the kidney.

Nitric oxide (NO) production and the NO synthases (NOS) are associated with multiple metabolic actions in the kidney that are important for normal renal function including regulation of renal hemodynamics and mediation of pressure natriuresis maintenance of medullary perfusion, blunting of tubule-glomerular feedback, inhibition of tubular sodium reabsorption and modulation of renal sympathetic neural activity. All three NOS isoforms, endothelial (eNOS), neuronal (nNOS) and inducible (iNOS) contribute to NO synthesis in kidney to maintain diuresis and natriuresis [18]. Renal I/R injury results in the generation of nitric oxide (NO) and causes multiple enzyme systems to be activated, including iNOS [19]. This causes cytoskeleton disruption, membrane damage and DNA degradation, and eventually cell death [20]. The activity of endothelial NOS (eNOS) is reduced during $\mathrm{I} / \mathrm{R}$ injury, possibly due to the high $\mathrm{NO}$ concentration generated by iNOS and this reduction in eNOS contributes to dysfunction and injury by promoting vasoconstriction of the renal circulation [19]. Alterations in the NO system are also associated with aging. In aged spontaneously hypertensive rats there are severe renal lesions and a NO deficiency with a decrease in renal eNOS compared to young rats [21]. In the renal arteries of older rats, there is an increase in iNOS compared to what is observed in younger rats [22]. Thus, both I/R injury and aging cause alterations in the NO system in the kidney. The combination of oxidative stress and alterations in the NO system results in the formation of increased levels of oxygen free radicals and reactive nitrogen species. NOS isoforms can act as sources of superoxide. All of these molecules can damage the mitochondria and cause cell death $[23,24]$. NO synthesized by eNOS plays an important role in maintaining renal blood flow following renal transplantation and therefore normal regulation of the NO system needs to be maintained [25]. Diabetic nephropathy is another kidney disease where oxidative stress is increased and NO production is altered. Dietary supplementation with antioxidants has been shown in several studies to have a positive effect on kidney function in diabetic nephropathy and these effects have been associated with changes in renal NO production and NOS activity [26-31] Because of the role of oxidative damage and free radicals in $\mathrm{I} / \mathrm{R}$ injury as well, pretreatment of scheduled donors with a diet fortified in antioxidants might help ameliorate free radical over-production and decrease I/R damage. Accordingly, the hypothesis of this study is that an antioxidant therapeutic treatment given to rats for a defined time both prior to and following renal I/R injury will reduce the kidney damage that results from I/R injury and improve renal function. The antioxidant diet used in this study was developed in our laboratories at Ohio University and successfully tested on a rat model of diabetic nephropathy [26]. The present study tests this antioxidant diet as a therapeutic approach to prevention of I/R injury.

\section{MATHERIALS AND METHODS}

\subsection{Animal Groups}

All experiments were conducted with the approval of the Animal Care and Use Committee of Ohio University. Studies were conducted on Wistar male rats $(n=31)$ obtained from Harlan-Sprague Dawley (Indianapolis, IN) at four weeks or 18 weeks of age, housed under controlled conditions of lighting, temperature and humidity. Starting at 6 weeks of age (younger animals) or upon arrival at 18 weeks of age (older animals), the rats were fed ad libitum a regular (REG) rat diet or a matched antioxidant-fortified (AO) diet, both from Purina Mills, Inc. (St. Louis, MO) as described before [26,27]. One week after the AO or REG diet was started, warm ischemia was induced and the respective diets continued for an additional week.

\subsection{Experimental Measurements}

In 8 week peri-pubertal rats and 20 week young adult rats, the following parameters were measured: body weight; kidney weight (KW); glomerular filtration rate (GFR), GFR adjusted to KW (GFR/KW) mean arterial pressure (MAP), urine NO and renal eNOS and iNOS in both cortex and medulla.

\subsection{Induction of 30 Minutes Warm Ischemia}

At 7 weeks or 19 weeks, rats were anesthetized with sodium pentobarbital $(60 \mathrm{mg} / \mathrm{kg}$, i.p., Abbott Laboratories, North Chicago, IL). A 30-minute period of pre-retrieval warm ischemia was achieved by intrinsic occlusion of the suprarenal aorta as described before. This model mimics a situation in which the donor kidney is exposed to low perfusion pressures and blood flow [32].

\subsection{Urinary NO Measurements}

Twenty-four hours prior to the experiment, animals were placed in metabolic cages and fasted, with free access to water. Urine collected for the 24 hours prior to the experiment was assayed for NO using the Apollo 4000 
free radical detector (World Precision Instruments, Sarasota, FL) [26].

\subsection{Kidney Function Measurements}

To assess the renal function 7 days post I/R injury, GFR was determined by the urinary iohexol clearance method using $180 \mathrm{mg} / \mathrm{ml}$ iohexol. The rat was anesthetized with ethyl-(1-methyl-propyl)-malonyl-thio-urea (Inactin; 100 $\mathrm{mg} / \mathrm{kg}$, i.p. Sigma-Aldrich Corporation, St. Louis, MO) and the procedure performed as described previously [26].

\subsection{Immunoblot Analyses of eNOS and iNOS}

Immunoblots were performed to assess the kidney cortex and medulla protein levels of the eNOS and iNOS as previously described [27].

\subsection{Statistical Analysis}

Results were compared between groups using one-way ANOVA and expressed as the mean \pm SEM. The 0.05 level of probability was used as the criterion of significance.

\section{RESULTS}

\subsection{Effect of Diet and Age on GFR}

The GFR was elevated above the normal range of $1.25 \pm$ $0.10 \mathrm{ml} / \mathrm{min}$ [33] at 8 weeks in animals on the REG diet exposed to I/R (Figure 1), while in the 8 week AO diet group the GFR was normal. There were no significant differences in GFR in $\mathrm{I} / \mathrm{R}$ rats with age. There were no differences in GFR/KW between groups on different diets or at different ages.

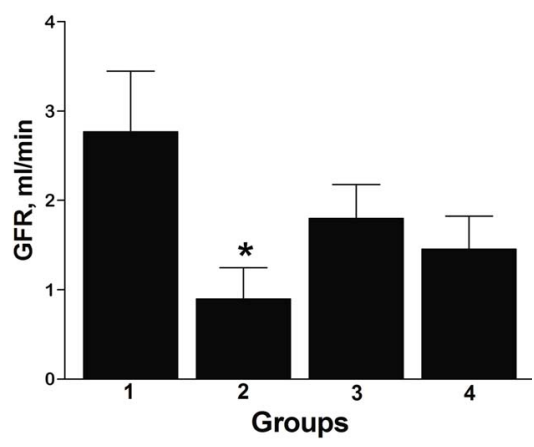

Figure 1. GFR according to the diet and age in I/R rats. Group 1: 8 weeks, REG diet; Group 2: 8 weeks, AO diet; Group 3: 20 weeks, REG diet; Group 4: 20 weeks, AO diet. ${ }^{*} \mathrm{p}<0.05$ between $\mathrm{AO}$ and REG diet, $\mathrm{n}=7$ (Group 1), 8 (Group 2), 8 (Group 3), 8 (Group 4). All data are shown as mean \pm SEM.

\subsection{Effect of Diet and Age on Urinary NO Excretion and MAP}

There were no statistically significant differences in urinary NO excretion related to the applied diet or age (Figure 2). There were no differences in MAP with age or diet (data not shown).

\subsection{Effect of Diet and Age on eNOS and iNOS Protein Content in Kidney Cortex and Medulla}

The obtained results of diet and age are presented for eNOS in Figure 3(a) and for iNOS in Figure 3(b). There were no significant effects of age observed in $\mathrm{I} / \mathrm{R}$ rats. In 8 week rats, levels of eNOS protein in cortex were higher on REG than on AO diet. There were no regional differences in NOS isoform content between kidney medulla and cortex. However, at 20 weeks, in rats on REG diet the amount of eNOS protein in cortex was higher than in medulla.

The immunoblots show a number of antibody-specific positive bands for each isoform that may represent alternatively spliced monomers and also dimers. For eNOS the bands detected were as follows: $380,320,280,254$, 210, 150, 116, 77, $67 \mathrm{kDa}$ that most likely represent homo- and hetero-dimers (380 - $210 \mathrm{kDa}$ ), splice forms of monomers (150 - $67 \mathrm{kDa})$ and differentially phosphorylated forms [34]. The iNOS bands were detected as follows: 322, 301, 224, 139, 122, 115, 77, $70 \mathrm{kDa}$ that most likely represent homo- and hetero-dimers (322 $224 \mathrm{kDa}$ ) and monomer splice forms (139 - $68 \mathrm{kDa})$.

In all groups of rats, the prevalence of monomers was higher than that of dimers (Figure 4). At 8 weeks the dimer/monomer ratios for eNOS in cortex were lower than at 20 weeks on the REG diet. At 20 weeks, the animals on the AO diet had a lower dimer/monomer ratio for eNOS in cortex than animals on the REG diet.

\section{DISCUSSION}

It is well established that oxidative stress is a contri-

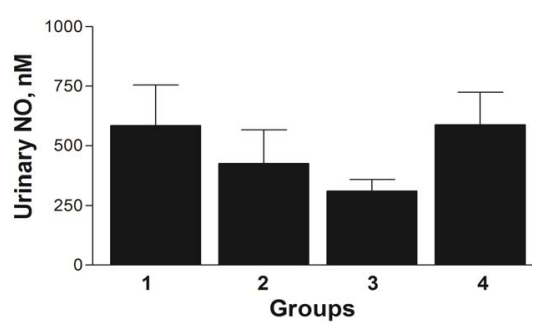

Figure 2. Effect of age and diet on urinary NO in I/R rats, Group 1: 8 weeks, REG diet; Group 2: 8 weeks, AO diet; Group 3: 20 weeks, REG diet; Group 4: 20 weeks, AO diet. $\mathrm{n}=5$ (Group 1), 6 (Group 2), 7 (Group 3), 2 (Group 4). All data are shown as mean \pm SEM. 


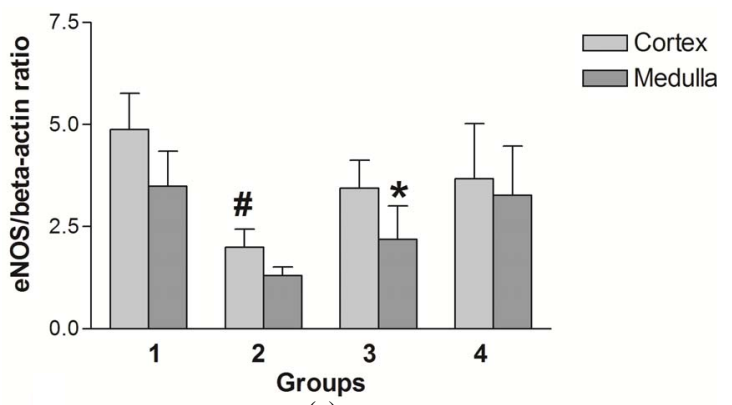

(a)

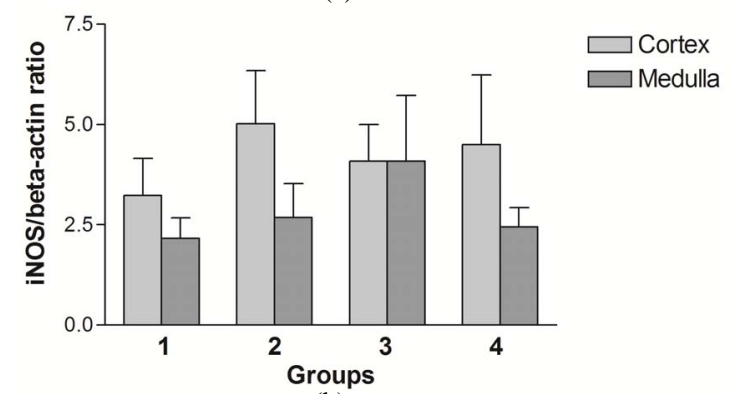

(b)

Figure 3. eNOS/beta-actin (a) and iNOS/beta-actin (b) ratios in I/R rat kidney cortex and medulla. Group 1: 8 weeks, REG diet; Group 2: 8 weeks, AO diet; Group 3: 20 weeks, REG diet; Group 4: 20 weeks, AO diet. For (a): $\mathrm{n}=7$ (Group 1), 2 (Group 2), 5 (Group 3), 3 (Group 4). For (b): $n=5$ (Group 1), 4 (Group 2), 3 (Group 3), 2 (Group 4). All data are shown as mean \pm SEM. ${ }^{*} p<0.05$ between REG and AO diet; ${ }^{*} \mathrm{p}<0.05$ between cortex and medulla.

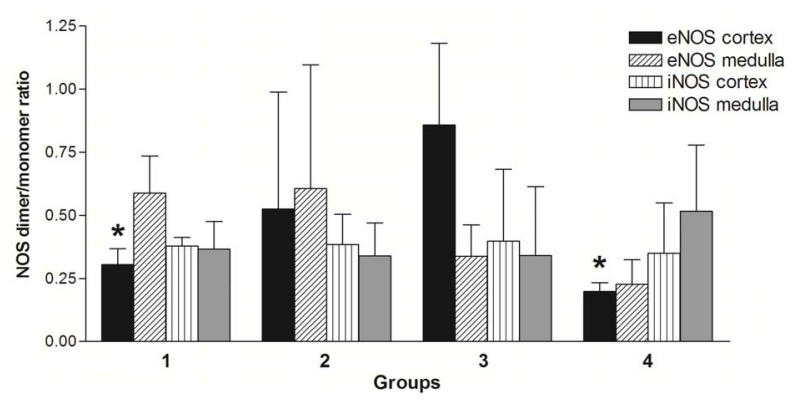

Figure 4. Effect of age and diet on eNOS and iNOS dimer/ monomer ratio in $\mathrm{I} / \mathrm{R}$ rats. Numbers of animals are the same as in Figures 3(a) and (b). All data are shown as mean \pm SEM. ${ }^{*} \mathrm{p}$ $<0.05$ compared to REG 20 week group.

buting factor to renal I/R injury [9-11]. The AO-fortified diet regimen used in the present study prevented early hyperfiltration in response to I/R injury, but only at 8 weeks of age. At 20 weeks, there was no difference in GFR between diets. Based on this, the AO-fortified diet may be more beneficial in very young animals and different $\mathrm{AO}$ formulations need to be tested to optimize the effect in more mature animals. We previously observed different responses with age to the diet used in this study, in obese diabetic Zucker rats with nephropathy $[26,27]$. The sensitivity to renal I/R injury also increases with age
[35], which may further exacerbate age-related differences.

Our data show a significant effect of the AO diet decreasing total eNOS protein at 8 weeks and a trend toward their being relatively more dimers in the AO diet group. Maintenance of normal or even elevated eNOS during ischemia and reperfusion has been shown to be beneficial to renal function. However, Increased oxidative stress can uncouple NOS, leading to a decrease in activity and some investigators have shown a decrease in eNOS activity despite an increase in protein in renal tubules early (24 - 72 hours) after I/R injury [19,36] leading to vasoconstriction. Data from the present study indicate higher levels of eNOS are associated with elevated GFR at 8 weeks (Group 1 vs Group 2, Figures 1 and 3(a)), at the later time interval of 1 week post injury. During this period, increased eNOS in renal cortex may result in renal vasodilation causing hyperperfusion and hyperfiltration, both of which damage the integrity of the glomerular capillary membrane, and may result in eventual decline in renal function [37]. Overall, this could reflect increased levels of functional eNOS dimers in the AO diet group in the setting of less oxidative stress, but increased total levels of eNOS contributing to increased levels of NO causing vasodilation in the hyperfiltering REG diet animals. An increase in eNOS, but not iNOS, in renal cortex is consistent with the findings of Veelken et al. who observed this in kidneys of diabetic rats during the early hyperfiltration phase of diabetic nephropathy [38].

Substantial elevations of NO can also contribute to formation of peroxynitrites and increased oxidative damage $[23,24]$. Although the urinary NO was higher in the REG diet males at 8 weeks compared to the AO diet males, this difference did not reach statistical significance, This may be due to the fact that urinary NO reflects the total circulating NO in addition to the renal NO. However, the increased filtration rate coupled with the increased NO concentration could result in increased NO excretion in the REG diet group.

In previous studies we showed a beneficial effect of this AO-fortified diet in the Zucker rat model of type 2 diabetes that was greater in females than their male counterparts $[26,27]$. Gender may also influence the severity of I/R injury due to the inhibitory effect of testosterone on eNOS activity [39]. It would thus be of interest to see how the AO diet affects female rats exposed to I/R injury.

The role of antioxidants and oxidative stress in $\mathrm{I} / \mathrm{R}$ injury is complex. Exposure to previous insults that increase oxidative stress in the kidney may result in subsequent protection by up-regulated endogenous factors such as superoxide dismutase [10]. In this setting, exogenous antioxidants may only be beneficial during cer- 
tain time intervals, possibly because of activation of other mechanisms of cellular injury and death, such as inflammation, increased intracellular calcium and activetion of caspases [7] by hypoxemia and acidosis.

Because dietary supplementation is a well-tolerated, cost effective therapy, future studies are needed that include additional modifications of dietary content and time frame, female gender, and older animals.

\section{AUTHORS CONTRIBUTIONS}

YS: Participated in writing of the paper, performance of the research, prepared the figures and performed statistical analysis of all data. FVN: Contributed substantially to writing of the manuscript and interpretation of the data, helped supervise performance of the experiments, contributed new reagents and analytical tools, participated in preparation of figures and data analysis. TMH: Participated in research design, performance of the research, and participated in data analysis. SRI: Participated in research design, writing of the paper, performance of the research, preparation of figures and data analysis.

\section{REFERENCES}

[1] Thiruchelvam, P.T., Willicombe, M., Hakim, N., Taube, D. and Papalois, V. (2011) Renal transplantation. BMJ, 343, d7300. doi:10.1136/bmj.d7300

[2] Kaisar, M.O., Nicol, D.L., Hawley, C.M., Mudge, D.W., Johnson, D.W., Preston, J.M., Wall, D.R., Griffin, A.D., Campbell, S.B. and Isbel, N.M. (2008) Change in live donor characteristics over the last 25 years: A single centre experience. Nephrology (Carlton), 13, 646-650. doi:10.1111/j.1440-1797.2008.01039.x

[3] Sahin, S., Manga Sahin, G., Turkmen, A. and Sever, M.S. (2006) Utilization of elderly donors in living related kidney transplantation. Transplantation Proceedings, 38, 385-387. doi:10.1016/j.transproceed.2005.12.090

[4] Stallone, G., Infante, B. and Gesualdo, L. (2010) Older donors and older recipients in kidney transplantation. Journal of Nephrology, 23, S98-S103.

[5] Gandhi, C., Zalawadia, R. and Balaraman, R. (2008) Nebivolol reduces experimentally induced warm renal ischemia reperfusion injury in rats. Renal Failure, 30, 921-930. doi:10.1080/08860220802353900

[6] Feng, L., Ke, N., Cheng, F., Guo, Y., Li, S., Li, Q. and Li, Y. (2011) The protective mechanism of ligustrazine against renal ischemia/reperfusion injury. Journal of Surgical Research, 166, 298-305. doi:10.1016/j.jss.2009.04.005

[7] Kosieradzki, M. and Rowinski, W. (2008) Ischemia/ reperfusion injury in kidney transplantation: Mechanisms and prevention. Transplantation Proceedings, 40, 3279-3288. doi:10.1016/j.transproceed.2008.10.004

[8] De Fijter, J.W. (2005) The impact of age on rejection in kidney transplantation. Drugs Aging, 22, 433-449. doi:10.2165/00002512-200522050-00007

[9] Nafar, M., Sahraei, Z., Salamzadeh, J., Samavat, S. and
Vaziri, N.D. (2011) Oxidative stress in kidney transplantation: Causes, consequences, and potential treatment. Iranian Journal of Kidney Diseases, 5, 357-372.

[10] Kim, J., Jang, H.S. and Park, K.M. (2010) Reactive oxygen species generated by renal ischemia and reperfusion trigger protection against subsequent renal ischemia and reperfusion injury in mice. American Journal of Physiology-Renal Physiology, 298, F158-F166. doi:10.1152/ajprenal.00474.2009

[11] Kim, J., Seok, Y.M. Jung, K.J. and Park, K.M. (2009) Reactive oxygen species/oxidative stress contributes to progression of kidney fibrosis following transient ischemic injury in mice. American Journal of PhysiologyRenal Physiology, 297, F461-F470. doi:10.1152/ajprenal.90735.2008

[12] Karaman, A., Turkmen, E., Gursul, C., Tas, E. and Fadillioglu, E. (2006) Prevention of renal ischemia/reperfusion-induced injury in rats by leflunomide. International Journal of Urology, 13, 1434-1441. doi:10.1111/j.1442-2042.2006.01592.x

[13] Bhalodia, Y., Sheth, N., Vaghasiya, J. and Jivani, N. (2010) Role of fenofibrate alone and in combination with telmisartan on renal ischemia/reperfusion injury. Renal Failure, 32, 1088-1094. doi:10.3109/0886022X.2010.504911

[14] Ozer Sehirli, A., Sener, G. and Ercan, F. (2009) Protective effects of pycnogenol against ischemia reperfusioninduced oxidative renal injury in rats. Renal Failure, 31, 690-697. doi:10.3109/08860220903085971

[15] Korkmaz, A. and Kolankaya, D. (2010) Protective effect of rutin on the ischemia/reperfusion induced damage in rat kidney. Journal of Surgical Research, 164, 309-315. doi:10.1016/j.jss.2009.03.022

[16] Chugh, G., Lokhandwala, M.F. and Asghar, M. (2011) Oxidative stress alters renal D1 and AT1 receptor functions and increases blood pressure in old rats. American Journal of Physiology_Renal Physiology, 300, F133F138. doi:10.1152/ajprenal.00465.2010

[17] Yang, H. and Fogo, A.B. (2010) Cell senescence in the aging kidney. Journal of the American Society of $\mathrm{Ne}$ phrology, 21, 1436-1439. doi:10.1681/ASN.2010020205

[18] Mount, P.F. and Power, D.A. (2006) Nitric oxide in the kidney: Functions and regulation of synthesis. Acta Physiologica, 187, 433-446. doi:10.1111/j.1748-1716.2006.01582.x

[19] Chatterjee, P.K. (2007) Novel pharmacological approaches to the treatment of renal ischemia-reperfusion injury: A comprehensive review. Naunyn-Schmiedeberg's Archives of Pharmacology, 376, 1-43. doi:10.1007/s00210-007-0183-5

[20] Lien, Y.H., Lai, L.W. and Silva, A.L. (2003) Pathogenesis of renal ischemia/reperfusion injury: Lessons from knockout mice. Life Sciences, 74, 543-552. doi:10.1016/j.lfs.2003.08.001

[21] Zhou, X.J., Vaziri, N.D., Zhang, J., Wang, H.W. and Wang, X.Q. (2002) Association of renal injury with nitric oxide deficiency in aged SHR: Prevention by hypertension control with AT1 blockade. Kidney International, 62, 
914-921. doi:10.1046/j.1523-1755.2002.00516.x

[22] Goettsch, W., Lattmann, T., Amann, K., Szibor, M., Morawietz, H., Munter, K., Muller, S.P., Shaw, S. and Barton, M. (2001) Increased expression of endothelin-1 and inducible nitric oxide synthase isoform II in aging arteries in vivo: Implications for atherosclerosis. Biochemical and Biophysical Research Communications, 280, 908-913. doi:10.1006/bbrc.2000.4180

[23] Huisman, A., Vos, I., van Faassen, E.E., Joles, J.A., Grone, H.J., Martasek, P., van Zonneveld, A.J., Vanin, A.F. and Rabelink, T.J. (2002) Anti-inflammatory effects of tetrahydrobiopterin on early rejection in renal allografts: Modulation of inducible nitric oxide synthase. FASEB Journal, 16, 1135-1137.

[24] MacMillan-Crow, L.A., Crow, J.P., Kerby, J.D., Beckman, J.S. and Thompson, J.A. (1996) Nitration and inactivation of manganese superoxide dismutase in chronic rejection of human renal allografts. Proceedings of the National Academy of Sciences of the USA, 93, 1185311858. doi:10.1073/pnas.93.21.11853

[25] Shoskes, D.A., Xie, Y. and Gonzalez-Cadavid, N.F. (1997) Nitric oxide synthase activity in renal ischemiareperfusion injury in the rat: Implications for renal transplantation. Transplantation, 63, 495-500. doi:10.1097/00007890-199702270-00002

[26] Slyvka, Y., Inman, S.R., Malgor, R., Jackson, E.J., Yee, J., Oshogwemoh, O., Adame, J. and Nowak, F.V. (2009) Protective effects of antioxidant-fortified diet on renal function and metabolic profile in obese Zucker rat. Endocrine, 35, 89-100. doi:10.1007/s12020-008-9121-7

[27] Slyvka, Y., Wang, Z., Yee, J., Inman, S.R. and Nowak, F.V. (2011) Antioxidant diet, gender and age affect renal expression of nitric oxide synthases in obese diabetic rats. Nitric Oxide, 24, 50-60. doi:10.1016/j.niox.2010.11.004

[28] Brigelius-Flohe, R. (2007) Adverse effects of vitamin E by induction of drug metabolism. Genes \& Nutrition, 2, 249-256. doi:10.1007/s12263-007-0055-0

[29] Douillet, C., Tabib, A., Bost, M., Accominotti, M., Borson-Chazot, F. and Ciavatti, M. (1996) A selenium supplement associated or not with vitamin E delays early renal lesions in experimental diabetes in rats. Proceedings of the Society for Experimental Biology and Medicine, 211, 323-331.

[30] Hamilton, S.J., Chew, G.T. and Watts, G.F. (2007) Therapeutic regulation of endothelial dysfunction in type
2 diabetes mellitus. Diabetes and Vascular Disease Research, 4, 89-102. doi:10.3132/dvdr.2007.026

[31] Montonen, J., Knekt, P., Jarvinen, R. and Reunanen, A. (2004) Dietary antioxidant intake and risk of type 2 diabetes. Diabetes Care, 27, 362-366. doi:10.2337/diacare.27.7.1845-a

[32] Inman, S.R., Plott, W.K., Pomilee, R.A., Antonelli, J.A. and Lewis, R.M. (2003) Endothelin-receptor blockade mitigates the adverse effect of preretrieval warm ischemia on posttransplantation renal function in rats. Transplantation, 75, 1655-1659. doi:10.1097/01.TP.0000063127.02261.E4

[33] Inman, S.R., Davis, N.A., Mazzone, M.E., Olson, K.M., Lukaszek, V.A. and Yoder, K.N. (2005) Simvastatin and L-arginine preserve renal function after ischemia/reperfusion injury. American Journal of the Medical Sciences, 329, 13-17. doi:10.1097/00000441-200501000-00003

[34] Mount, P.F., Kemp, B.E. and Power, D.A. (2007) Regulation of endothelial and myocardial NO synthesis by multi-site eNOS phosphorylation. Journal of Molecular and Cellular Cardiology, 42, 271-279. doi:10.1016/j.yimcc.2006.05.023

[35] Kusaka, J., Koga, H., Hagiwara, S., Hasegawa, A., Kudo, K. and Noguchi, T. (2012) Age-dependent responses to renal ischemia-reperfusion injury. Journal of Surgical Research, 172, 153-158. doi:10.1016/j.jss.2010.08.034

[36] Vinas, J.L., Sola, A., Genesca, M., Alfaro, V., Pi, F. and Hotter, G. (2006) NO and NOS isoforms in the development of apoptosis in renal ischemia/reperfusion. Free Radical Biology \& Medicine, 40, 992-1003. doi:10.1016/j.freeradbiomed.2005.10.046

[37] Moudgil, A. and Bagga, A. (1999) Evaluation and treatment of chronic renal failure. Indian Journal of Pediatrics, 66, 241-253. doi:10.1007/BF02761215

[38] Veelken, R., Hilgers, K.F., Hartner, A., Haas, A., Bohmer, K.P. and Sterzel, R.B. (2000) Nitric oxide synthase isoforms and glomerular hyperfiltration in early diabetic nephropathy. Journal of the American Society of Nephrology, 11, 71-79.

[39] Park, K.M., Kim, J.I., Ahn, Y., Bonventre, A.J. and Bonventre, J.V. (2004) Testosterone is responsible for enhanced susceptibility of males to ischemic renal injury. The Journal of Biological Chemistry, 279, 52282-52292. doi:10.1074/jbc.M407629200 